\title{
Human Emotion Recognition Using Mean of Average and Maximum Pooling
}

\author{
C.Karthik, D.Chandrasekhar, B.Naveen Kumar, B.Naveen
}

\begin{abstract}
Currently a the very beginning's of the unsolved difficulty in pc imaginative and prescient is perceiving or understanding different people' feelings and sentiments. Albeit ongoing strategies accomplish close to human exactness in controlled conditions, the acknowledgment of emotions inside the wild remains a hard difficulty. On this paper we proposed MAM Pooling (mean of common and maximum) strategy with CNN to perceive human feelings. We center round programmed distinguishing evidence of six emotions constantly: Happiness, Anger, unhappiness, surprise, fear, and Disgust. Convolutional Neural network (CNN) is a certainly propelled trainable layout that may study invariant highlights for numerous programs. Whilst all is said in carried out, CNNs include of rotating convolutional layers, non-linearity layers and highlight pooling layers. In this artwork, a

Novel include pooling approach, named as MAM pooling is proposed to regularize CNNs, which replaces the deterministic pooling obligations with a stochastic system thru taking the ordinary of max pooling and regular pooling strategies. The benefit of the proposed MAM pooling technique lies in its firstrate capability to address the over fitting problem skilled with the resource of CNN age.
\end{abstract}

Keywords-Emotion; Face Expression; MAM Pooling, CNN.

\section{INTRODUCTION}

Because of the fact its first advent inside the mid 1980's [1], the Convolutional Neural community (CNN) has displayed grand suggests for particular packages; for example, composed with the resource of hand digit confirmation [2], face acknowledgment [3], and so forth. With the advances of fake reputation, overdue years have seen the creating commonness of big gaining knowledge of with CNNs on steadily stuck seen acknowledgment responsibilities. In [4], Fan et al. View human after as a mastering difficulty of assessing the territory and the size of factors and use CNNs to accomplish this studying reason. Cire, san et al. [5] endorse a plan of multi-fragment CNNs which can be lively via images Processing Unit (GPU) for image course of motion and stunning displays are cultivated on one-of-a-kind benchmark datasets. In [6], a 3-d CNN show is deliberate for human movement confirmation, wherein both the spatial and quick capabilities are mined by means of performing 3-D convolutions. Krizhevsky et al. [7]

Revised Manuscript Received on September 14, 2019.

C.Karthik, Associate Prof, Department of ECE , QIS Group Colleges, Ongole, Andhra Pradesh, India. (E-mail:

D.Chandrasekhar, Assist. Prof, Department of ECE, Siddhartha Institute of Technology \& Sciences, Narapally, Ghatkesar, Hyderabad, Telangana, India..

B.Naveen Kumar` Assist. Prof, Department of ECE, Siddhartha Institute of Technology \& Sciences, Narapally, Ghatkesar, Hyderabad, Telangana, India..

B.Naveen, Assist. Prof, Department of ECE, Siddhartha Institute of Technology \& Sciences, Narapally, Ghatkesar, Hyderabad, Telangana, India.. karthik.kamalesh@gmail.com)

set up a top notch CNN for the ImageNet visible confirmation venture [8] and achieve a shocking recordbreaking execution in 2012. However the presently referenced appealing advances, there are up 'til now a couple of issues experienced via way of CNNs, as an instance, the over-becoming hassle in view of the immoderate furthest reaches of CNNs. With a particular closing goal to deal with this problem, more than one regularization methodologies had been proposed, as an instance, weight decay, weight tying and increment of getting organized devices [9]. Those regularization techniques permit the education of bigger restriction models than would possibly by means of some method be potential, that may accomplish traditional check exhibitions as contrasted and littler un-regularized models [10]. Another promising regularization method is Dropout this is proposed through Hinton et al. [11]. The possibility of Dropout is to stochastically set an good sized phase of the establishments in a covered layer to zeros for each making plans take a look at. With the aid of doing this, the protected devices cannot co-change according with each other, and they must absorb a higher depiction for the commitment than solicitation to summarize well. Dropout acts like a form of version averaging over every viable release of the model, and it's miles regarded to bypass on basic receives in execution in specific programs. Anyhow, the inability of Dropout is that it cannot be typically used for a couple of styles of CNN layers, for example, the convolutional layer, non-linearity layer and feature pooling layer. To beat this sickness, a speculation of Dropout, known as DropConnect, is proposed in [12]. In vicinity of haphazardly selecting initiations within the device, DropConnect devices an arbitrarily decided on subset of hundreds to zeros. While contrasted with Dropout, better exhibitions have been finished by using DropConnect in specific instances. In [10], some other kind of regularization for convolutional layers, named stochastic pooling, is proposed to have interaction the arrangement of more fashions for weakening overbecoming. The important thing concept of stochastic pooling is to effect the pooling to manner in each convolutional layer a stochastic method thinking about multinomial dissemination. In this art work, as [10], a singular form of pooling approach, named as mixed pooling, is proposed an amazing way to aid the regularization execution for making geared up larger CNN fashions. Enlivened via Dropout (that haphazardly units a huge a part of the enactments to zeros), the proposed MAM pooling technique replaces the standard deterministic pooling sports with a stochastic technique stochastic device thru taking the 
normal of max pooling and normal pooling strategies at a few stage inside the coaching of CNNs. This form of stochastic nature of the proposed MAM pooling approach averts over-turning into extraordinarily. Examinations are executed to verify the superiority of the proposed MAM pooling strategy over the normal max pooling and regular pooling techniques.

\section{WRITING SURVEY/RELATED PAINTINGS}

A short evaluation of $\mathrm{CNNs}$ is displayed therefore which is big to evoke the proposed combined pooling technique. Whilst all is said in completed, CNNs are dealers of the multi-set up Hubel-Wiesel engineering [13], which get rid of nearby functions at a excessive assure except, logically be part of these into step by step unpredictable features at cut down dreams. The dearth of spatial facts is reimbursed through a growing quantity of function maps in higher layers.

A proficient $\mathrm{CNN}$ is produced from multiple issue extraction tiers, and every set up consists of a convolutional layer, a non-straight trade layer and an consist of pooling layer. The convolutional layer takes inward aftereffect of the right away channel and the important open issue took after through the use of a nonlinear sanctioning artwork at every close by fragment of the statistics. Through then, the nonwithout delay change layer plays institutionalization among near to feature maps. In the end, the factor pooling layer joins close by neighborhoods the usage of an normal or maximum high-quality motion, hoping to gather invariance to little turns. An instance of a -type out CNN with the these days referenced three layers is confirmed up in Fig. 1 for portrayal.

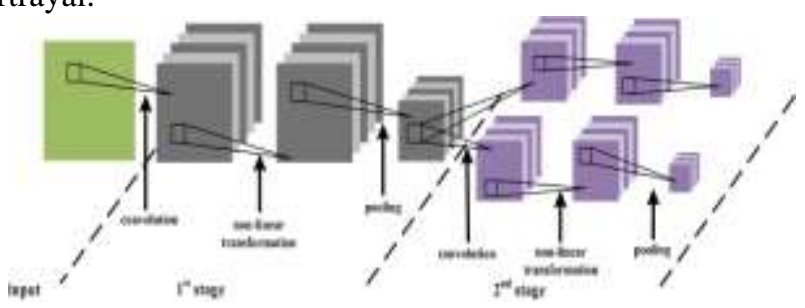

Figure 1. A two-set case CNN. A picture data through a convolutional layer, deserted a layer of non-direct change and pooling layer for low-level discharge features on the primary organize. Around then, the three layers are associated again in the subsequent stage to isolate feature anomalous state.

\section{DEPICTION OF THE PROPOSED APPROACH}

\section{A. Convolutional Layer}

Point convolutional layer is to isolate cases found in the close by locale of pictures information is exceptionally ordinary in the general picture [10]. For the most part, convolutional layer producing map highlights with direct convolution channel pursued by a nonlinear enactment work, for example, ReLU [14], sigmoid, tanh, and so on. In this vein, the yield yk k map highlights can be determined as pursues:

$$
\mathrm{yk}=\mathrm{f}(\mathrm{wk} * \mathrm{x})-----------(1)
$$

Where $\mathrm{x}$ denotes the input image, wk stands for convolutional channel related with map highlights $\mathrm{k}$, * means the 2D convolution administrator which is utilized to compute the inward item format channel at every area in the information picture, and $f(\bullet)$ is a nonlinear actuation work.

\section{A. Non-straight change layer}

It has been appeared in [15] utilizing redressing nondirect change layer is a ground-breaking technique to extra improve execution $\mathrm{CNN}$ for visual acknowledgment undertakings. This layer is commonly perform assignments subtractive or upset close by for institutionalization, executing the nearest kind of rivalry among the features in a similar format on the different guide parts. Typically there are two sorts of non-straight changes. One is the reaction of neighborhood standardization [11], which produces yield ykij standardized at the position $(\mathrm{I}, \mathrm{j}) \mathrm{k}$ as a component mapy_kij "=" (x_kij $) / \llbracket\left[1+\alpha / \mathrm{N} . \sum(\mathrm{l}=\mathrm{k}-\mathrm{N} / 2)^{\wedge}(\mathrm{k}+\mathrm{N} / 2)\right.$ w.: $\llbracket \llbracket \llbracket\left(x \rrbracket \_\right.$lij $\left.) \rrbracket \wedge(2)\right] \rrbracket \rrbracket \wedge \beta \quad---(2)$

\section{B Feature Pooling Layer}

The inspiration driving pooling is to change the delineation of the components together into one longer applied that records is remarkable even as arranging jam giant focal points. Pooling layer works in $\mathrm{CNNs}$ plans to perform invariant to modifications in position or lights conditions, the capability to the chaos, and the minimization of delineation. At the element whilst all is said in completed, pooling layer depicts the consequences of the gathering neighboring neurons in a lessen of a comparable line. In pooling layer, the guarantee map component diminished via fusing grade by grade ecological nearby on map very last layer phase, alongside those strains expanding the invariant for the mutilation of the belongings.

In CNNs, there are everyday pooling system, which encompass pooling max further, pooling wellknown. Most extreme technique pooling choosing the largest phase in every close by pooling as

$$
\text { y_kij } "="\left(\_(p, q) \in R \_i j \wedge(\max )\right) \quad \square \quad x \rrbracket \_k p q--(3)
$$

where ykij is the output of the pooling operator related to the kth feature map, xkpq is the element at $(p, q)$ within the pooling region $\mathrm{Rij}$ which represents a local neighborhood around the position ( $i, j$ ). Regarding the average pooling method, it takes the arithmetic mean of the elements in each pooling region a

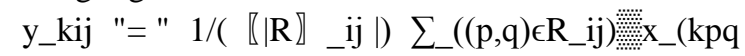

\section{$B$ Proposed MAM (Mean of Average and Maximum)} Pooling

As referenced earlier than, pooling $\max$ and regular association of two strategies used by CNNs a famous selection in light in their computational effectiveness. for instance, the regular pooling technique applied in [15] who got top notch

photo arrangement precision on Caltech101 dataset. In 
[7], max pooling method changed into effectively implemented to prepare in 'convnet' for ImageNet contention. Albeit the two styles of pooling administrator can characteristic admirably

In some datasets, it's far as yet obscure who will work higher to take care of latest issues. as it have been, it is a kind of induction to choose pooling administrator.

on the other hand, each the maximum severe pooling and pooling usual directors have their very own particular shortcomings. about max pooling, it just considers the maximum outrageous segments and others in the vicinity confronting pooling. now and again, it will ask inadmissible consequences. as an instance, if most of the parts inside the sector of the degree of pooling is excessive, tops vanish after max pooling perceive as suggests up in Fig. 2 (a). For the association, it computes the suggest of an sizable wide variety of segments in the surveying vicinity. This manager will take all degrees low to instinct and new pooling Delineate separation elements could be faded. Shockingly gradually horrible, if there are many zero parts, typical to the manual thing might be decreased, all matters considered, as alluded to in Fig. 2(b)
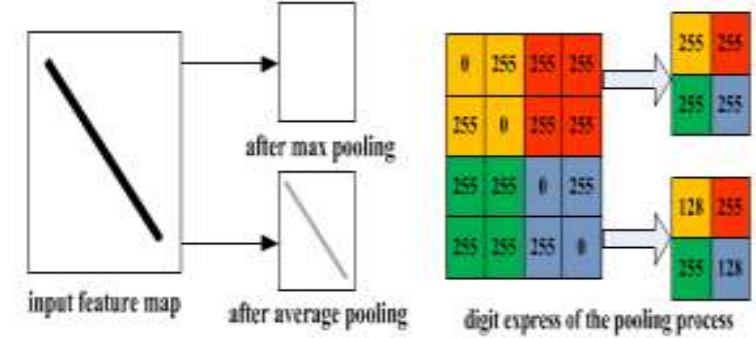

(a) Illustration of max pooling drawbach

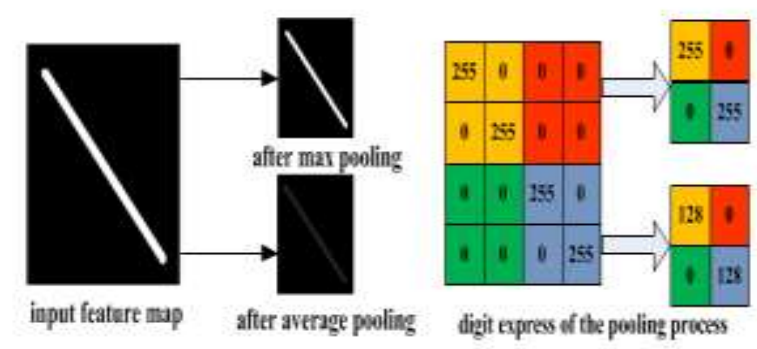

(b) Illustration of average pooling drawback

Fig. 2. Toy example illustrating the drawbacks of max pooling and average pooling.

It ought to be noticed that the pictures in the regular world is reliably changing, and it is preposterously high that damaged pieces of max pooling and ordinary unification, (for example, showing up in Fig. 2) will have a negative sway in applying the covering to CNNs pooling. Thusly, as an answer, we consider supplanting the deterministic pooling movement with stochastic strategies, which use close by self-assertive max pooling and ordinary association methodology while planning CNNs. It is recommended that blended pooling technique that will be displayed straightforwardly.

\section{Pooling Scheme:}

MAM proposed pooling roused by irregular Dropout [11] and Drop-Connect [12] strategy. As referenced before, when preparing with Dropout, a haphazardly chosen subset of enactment are set to focus in each layer. Concerning DropConnect, it's not set an arbitrarily chosen subset of loads in the system to zero. Both of these two methods have demonstrated to be solid for neural arrange settings. In this work, the proposed pooling MAM strategy delivers the yield is gathered by the accompanying recipe.

$$
\text { y_kij "=" }\left(\left(\_(p, q) \in R_{-} i^{\wedge}(\max )\right) \llbracket \mathrm{x} \rrbracket \_k p q+1 /(\llbracket\right.
$$

$\left.\left|R \rrbracket \_i j\right|\right) \quad \sum\left((p, q) \in R \_i j\right)$ w:

Where $\lambda$ is a random value being either 0 or 1 , indicating the choice of using the max pooling or average pooling. In another word, the proposed method changes the pooling regulation scheme in a stochastic manner which will address the problems encountered by max pooling and average pooling to some extent.

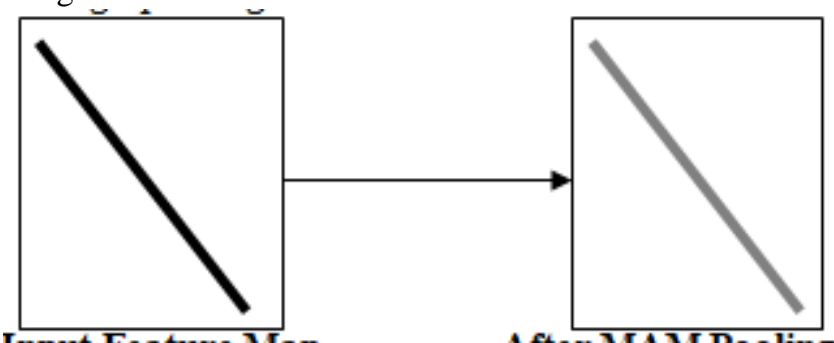

Input Feature Map

After MAM Pooling

FIG3(a): The drawback of max is overcome by MAM Pooling
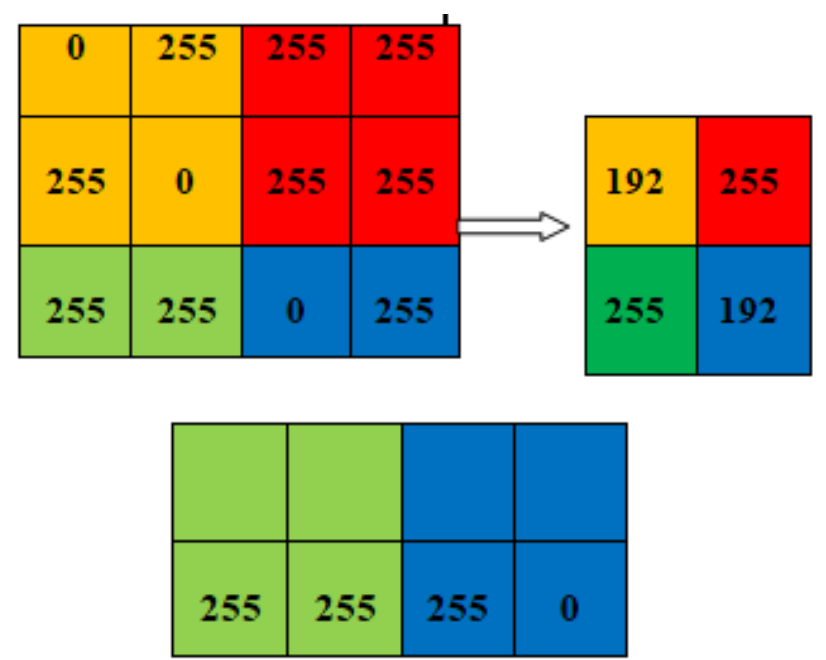

FIG3(b): The drawback of max is overcome by MAM Pooling

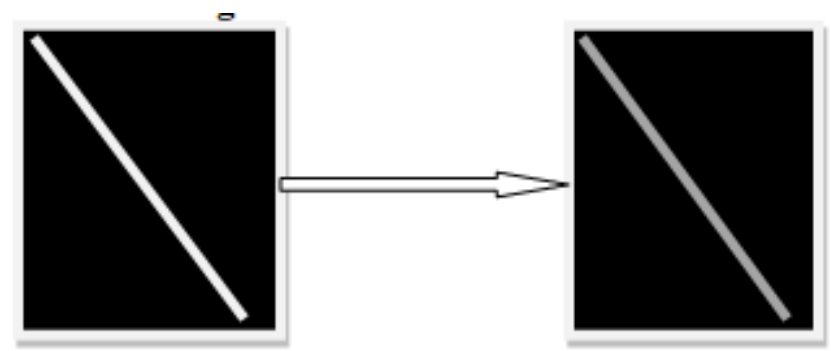

Input Feature Map

After MAM Pooling

FIG4(a): The drawback of average is overcome by MAM Pooling 


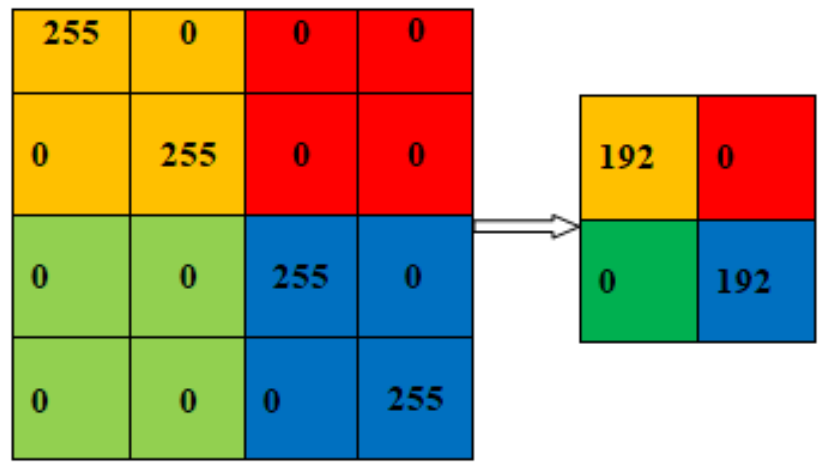

Fig4(B): The Drawback of Max is Overcome By Mam
Pooling

\section{RESULTS}

In this work, an extensive experimentation with cross data base facial expression recognition is presented and the influence of fine-tuning a CNN pre-trained for a different task in a similar domain (face recognition) is investigated. By using this MAM pooling, we got more than $98 \%$ human emotions recognition using facial expressions.

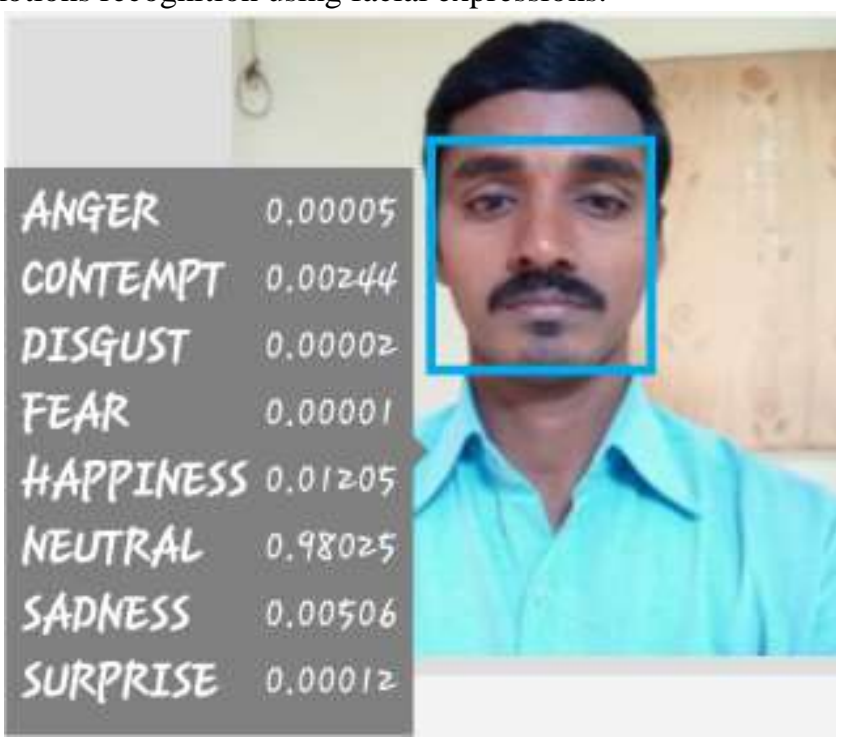

FIG5: Human emotion recognition using MAM pooling based convolutional neural networks

\section{CONCLUSION}

On this paper, every other association device referred to as MAM proposed pooling, which can be the approach by taking the ordinary of the everyday pooling and pooling process max. Similar exams passed off pooling MAM suggests that the proposed technique is superior to some thing the traditional pooling max normal and pooling systems to defeat the issue of over-becoming and enhance the accuracy portrayal. With our proposed device of increasing human feelings exactly. Which likewise offers greater solidarity to $\mathrm{CNN}$ in pooling level?.

\section{REFERENCES}

1. Fukushima, ok. Neocognitron: A model of self-finding out neural structures for design acknowledgment aspect is caused by a circulate in position. Natural Cybernetics 36 (4), 193-202 (1980)
2. LeCun, Y., Boser, B., Denker, J.S., Henderson, D., Howard, R.E., Hubbard, W.Jackel, L.D.: Backpropagation implemented to penmanship acknowledgment postal division. Nerve Estimation 1 (four), 541-551 (1989)

3. Lawrence, S., Giles, C.L., Tsoi, A.C., once more, A.D.: Face acknowledgment: convolutional neural-set up approach. IEEE Transactions on Neural Networks eight (1), ninety eight-113 (1997)

4. Fan, J., Xu, W., Wu, Y., Gong, Y.: Human following using convolutional neural device. IEEE Transactions on Neural Networks 21 (10), 1610-1623 (2010)

5. Cire,san, D., Meier, U., Schmidhuber, J.: Multi-phase in a neural machine for photograph grouping. In: CVPR, pp 3642-3649 (2012).

6. Ji, S., Xu, W., Yang, M., Yu, ok.: 3-D convolutional neural gadget for the acknowledgment of human interest. IEEE Transactions on sample assessment and tool Intelligence 35 (1), 221-231 (2013)

7. Krizhevsky, A., Sutskever, I., Hinton, G.E.: ImageNet grouping with neural structures in the convolutional. In NIPS, vol. 1 (2012)

8. Deng, J., Dong, W, Socher, R., Li, L.- J., Li, good enough., Fei-Fei, L.: ImageNet :. A massive scale severa leveled image database. In: CVPR, pp 248-255 (2012).

9. Montavon, G., Orr, G.B., Muller, okay.R. (Eds.): Neural systems: little-acknowledged techniques, 2 model. Spinger, San Francisco (2012)

10. Zeiler, M.D.: Hierarchical convolutional profound learning in pc vision. $\mathrm{PhD}$ proposition, ch. 6 , the big apple university (2014)

11. Hinton, G.E., Srivastava, N., Krizhevsky, A., Sutskever, I., Salakhutdinov, R.R.:enhancing neural structures by co-adjustment reventing locator highlights. ArXiv preprint arXiv: 1207.0580 (2012)

12. Wan, L., Zeiler, M.D., Zhang, S., LeCun, Y., Fergus, R.: Regularization of neural structures the use of DropConnect. In: ICML, pp 1058-1066 (2013).

13. put together LeCun, Y., Kavukcuoglu, k., Farabet, C.: Convolutional and programs in imaginative and prescient. In: ISCAS, pp 253-256 (2010).

14. Nair, V., Hinton, G.E.: Rectified advanced direct unit is restrained Boltzmann tool. In: ICML, pp 807-814 (2010).

15. Jarrett, okay., Kavukcuoglu, okay., Ranzato, M., LeCun, Y.:what's the pleasant multi-prepare engineering for item acknowledgment? In: ICCV, pp 2146-2153 (2009). 